\title{
Captura de carbono en sistemas agroforestales en el Perú
}

\author{
Carbon capture in agroforestry systems in Peru
}

\author{
Edith R. Clemente-Arenas ${ }^{1, *}$
}

Recibido: 05 noviembre 2020|Aceptado: 02 diciembre 2021|Publicado en línea: 29 diciembre 2021

Citación: Clemente-Arenas, ER. 2021. Captura de carbono en sistemas agroforestales en el Perú. Revista Forestal del Perú 36(2): 180-196. DOI: http://dx.doi.org/10.21704/rfp.v36i2.1797

\begin{abstract}
Resumen
Los sistemas agroforestales han sido reconocidos mundialmente desde hace unas décadas atrás por sus beneficios económicos y de conservación de suelo, agua y biodiversidad. En el Perú esta técnica se está extendiendo principalmente en la Amazonía, con el uso del componente forestal que brinda sombra a los principales cultivos de exportación como café y cacao, entre otros frutales. Sin embargo, los sistemas agroforestales representan muchos beneficios económicos y ecológicos, como la conservación de las propiedades del suelo, conservación de la biodiversidad, agua y aire. La importancia de los sistemas agroforestales en la captura de carbono se resume en dos conceptos: el primero es que los árboles se convierten en sumideros de carbono a largo plazo al capturar carbono mediante la fotosíntesis y el segundo es que reducen la necesidad de deforestar nuevos bosques para una agricultura migratoria deficiente. El propósito de esta revisión es analizar el potencial de la captura de carbono en los sistemas agroforestales en el Perú para lograr una agricultura sostenible económica y ecológicamente con esta técnica agroforestal. La información obtenida refleja mediciones en centros de experimentación y parcelas de agricultores en regiones de la amazonia peruana como: Huánuco, San Martin, Ucayali, Loreto, Pasco, Amazonas, Madre de Dios y Puno. Estos estudios mayormente presentan resultados de evaluaciones de stock de carbono, mas no de captura de carbono como lo indica el título, la captura de carbono debe ser evaluado durante un periodo de tiempo para obtener la cantidad de carbono capturado de la atmosfera en una unidad de espacio y por una unidad de tiempo ( $\mathrm{Mg} / \mathrm{ha} / \mathrm{año})$. Resaltan los sistemas agroforestales de Huánuco y Loreto, el primero con un stock de carbono de $344,24 \mathrm{Mg} / \mathrm{ha}$ en un sistema agroforestal de cacao (Theobroma cacao L.) y otras especies forestales de más de 16 años y el segundo con $288,98 \mathrm{Mg} / \mathrm{ha}$ en un sistema agroforestal de castaña (Bertholletia excelsa Bonpl.), umari (Poraqueiba sericea Tul.), tornillo (Cedrelinga cateniformis (Ducke) Ducke) y pashaco (Senna multijuga (Rich.) H.S. Irwin \& Barneby) sin edad conocida. Además, existe una falta de estandarización de metodologías en la estimación de carbono sobre y debajo del suelo, para poder realizar comparaciones y estudios a una mayor escala. Concluimos que los sistemas agroforestales representan la mejor opción en el secuestro de carbono después de los bosques naturales y presentan una tecnología económica y ecológicamente sostenible, la cual se
\end{abstract}

${ }^{1}$ Estación Experimental Agraria San Bernardo - Instituto Nacional de Innovación Agraria (INIA), Madre de Dios, Perú.

*Autor de Correspondencia: edith-clemente-a@outlook.com 
busca en la actualidad para evitar los efectos de la deforestación y el calentamiento global. Proponemos a estos sistemas como solución frente al avance de tierras degradadas por la agricultura tradicional de corte y quema, procurando mayor investigación de todos sus aspectos y componentes, para dilucidar los procesos elementales que impulsan o limitan el uso de los sistemas agroforestales.

Palabras clave: sumidero de carbono, Amazonía, agroforestería

\begin{abstract}
Agroforestry systems have been recognized worldwide for decades for their economic benefits and conservation of soil, water and biodiversity. In Peru, this technique is spreading mainly in the Amazon, with the use of the forest component that provides shade for the main export crops such as coffee and cocoa, among other fruit trees. However, agroforestry systems represent many economic and ecological benefits, such as the conservation of soil properties, conservation of biodiversity, water and air. The importance of agroforestry systems in carbon sequestration is summarized in two points: the first is that trees become long-term carbon sinks by capturing carbon through photosynthesis and the second is that they reduce the need to deforest new forests for poor shifting cultivation. The purpose of this review is to analyze the potential of carbon sequestration in agroforestry systems in Peru to achieve economically and ecologically sustainable agriculture with this agroforestry technique. The information obtained reflects measurements in experimentation centers and farmers' plots in regions of the Peruvian Amazon such as: Huánuco, San Martin, Ucayali, Loreto, Pasco, Amazonas, Madre de Dios and Puno. These studies mostly present evaluations of carbon stock and not carbon capture as indicated, which must be evaluated over a period of time to obtain the amount of carbon captured from the atmosphere in a unit of space and per unit of time $(\mathrm{Mg} /$ ha/year). The agroforestry systems of Huánuco and Loreto stand out, the first with a carbon stock of $344.24 \mathrm{Mg} / \mathrm{ha}$ in an agroforestry system of cocoa (Theobroma cacao L.) and other forest species over 16 years old and the second with $288.98 \mathrm{Mg} / \mathrm{ha}$ in an agroforestry system of Brazil-nut (Bertholletia excelsa Bonpl.), umari (Poraqueiba sericea Tul.), tornillo (Cedrelinga cateniformis (Ducke) Ducke) and pashaco (Senna multijuga (Rich.) H.S. Irwin \& Barneby) with no known age. In addition, there is a lack of standardization of methodologies in the estimation of carbon above and below the ground, to be able to carry out comparisons and studies on a larger scale. We conclude that agroforestry systems represent the best option in carbon sequestration after $n$ atural forests and present a sustainable economic and ecological technology, which is currently being sought to avoid the effects of deforestation and global warming. We propose these systems as a solution to the advance of lands degraded by traditional slash-and-burn agriculture, seeking further investigation of all its aspects and components, to elucidate the elemental processes that drive or limit the use of agroforestry systems.
\end{abstract}

Key words: carbon sink, Amazon, agroforestry

\section{Introducción}

Los sistemas agroforestales (SAFs) son una alternativa productiva sostenible que consiste en una práctica de uso de suelo, donde se combinan especies leñosas perennes con cultivos y/o ganado en un mismo espacio logrando un eficiente uso de los recursos como nutrientes del suelo, luz y agua; con el propósito funda- mental de diversificar y optimizar la productividad teniendo en cuenta el principio de sostenibilidad (Garrett et al. 2009).

Analizando conceptos, vemos que los SAFs comparados con los monocultivos tienen como principal característica a su diseño, el cual le brinda una mayor diversidad estructural, con una disposición horizontal del compo- 
nente leñoso, el cual puede ser mezclada o zonal con respecto al cultivo (Nair 2011). Mientras que la disposición vertical en los SAFs es multiestratificada lo que favorece la captación de luz para los cultivos que suelen ser tolerantes a la sombra, aprovechando los estratos superiores con especies con alto requerimiento de luz. Esta diversidad estructural permite conservar los ciclos de nutrientes más cerrados, por lo tanto, estos sistemas presenta una mayor estabilidad y resistencia a nivel local, junto con una alta conectividad entre los bosques y otros paisajes a nivel de paisaje y cuenca (Nair y Garrity 2012). Cultivar una mezcla de árboles y arbustos de esta manera imita el comportamiento de un bosque y ayuda al suelo a retener nutrientes y agua, protegiendo la tierra y aumentando la absorción de carbono.

Dentro del SAF las especies leñosas tienen como función principal, mantener o mejorar la productividad del sistema mediante la protección de los cultivos del intenso calor y de las lluvias, disminuir la evapotranspiración y aumentar el ciclaje de nutrientes (Gold y Garrett 2009). Adicionalmente, en un sistema agroforestal se obtienen otros productos y servicios (frutas, madera, leña, plantas aromáticas y medicinales, acumulación de $\mathrm{CO}_{2}$, liberación de oxígeno, conservación del suelo, diversificación del paisaje, alimento y refugio de fauna silvestre) (Beer et al. 2003, Casanova-Lugo et al. 2016). El aprovechamiento de la luz, humedad y nutrientes del suelo constituyen los secretos del manejo eficiente de esta tecnología agroforestal.

La característica principal de los SAFs es de proveer diferentes productos y a la vez brindar servicios ambientales similares o incluso superiores que los ecosistemas naturales. En el ámbito económico los sistemas agroforestales representan un ahorro potencial de capital, porque generarán ganancias a largo plazo, esto debido a la inversión en los árboles madereros, que podrían reducir las pérdidas financieras causadas por las caídas repentinas en los precios de comercialización de los cultivos del SAF (Tscharntke et al. 2011). Además, las características que describen a los SAFs proporcionan muchos otros beneficios como: la conser- vación de las propiedades del suelo donde los árboles pueden mejorar las condiciones físicas, químicas y biológicas de los suelos. Físicamente mediante la cubierta protectora del suelo que provee la hojarasca y el dosel de los árboles de la erosión hídrica y la desecación por los rayos solares; por otro lado, las raíces profundas de los árboles ayudan a mejorar textura del suelo dándole una mayor porosidad, evitando su compactación y aumenta la tasa de infiltración del agua (Allen et al. 2004). Químicamente, los árboles incorporan materia orgánica a los suelos en forma de hojarasca, favoreciendo los ciclos de nutrientes (Petit-Aldana et al. 2012). Biológicamente, los suelos de los sistemas agroforestales conservan la diversidad de microorganismos y aseguran su fertilidad mediante la capacidad y velocidad de descomposición de la materia orgánica en los ciclos de nitrógeno y carbono. Por otro lado, los SAFs dentro de los paisajes deforestados y fragmentados suministran hábitats y recursos para las especies de plantas y animales; mantienen la conexión del paisaje mediante corredores entre los remanentes de los hábitats; y evitan la degradación y perdida de hábitats (Schroth y Sinclair 2003, Shibu 2009). Además, intervienen en la conservación del agua debido a que los árboles influyen en el ciclo del agua por que incrementan la intercepción de la lluvia y de nubes e igualmente modifican la transpiración y aumentan la retención de agua en el suelo porque reducen la escorrentía e incrementan la infiltración (Beer et al. 2003).

En el Perú, los SAFs se han desarrollado desde tiempos antiguos en las tribus indígenas de la amazonia, los cuales practicaban la agricultura rotacional. Esto consistía, primero en una tala y quema de los bosques, seguido de la siembra de cultivos anuales y semi-perennes. Después de un periodo de tiempo de descanso de la tierra que pueden ser meses o años se procede con la siembra intercalada o sucesiva de cultivos perennes, donde la intensidad disminuye a lo largo de los años. En un inicio se realiza un frecuente deshierbe y progresivamente disminuye su mantenimiento hasta una eventual regeneración de crecimiento en gran parte natural (Padoch et al. 1985). La agricultura migratoria 
ha generado una preocupante deforestación en el Perú que se ha ido incrementando en las últimas décadas, con una tasa de deforestación anual de $830 \mathrm{~km}^{2}$ en 2001, mientras que en 2014 supero los $1770 \mathrm{~km}^{2}$; por lo que se estima que para el 2030 está tasa supere los $3500 \mathrm{~km}^{2}$ (MINAM 2016). Esto afecta principalmente la alta diversidad que albergan estos bosques, principalmente en departamentos como San Martin (19,42\%), Loreto (14,68\%) y Amazonas (12,30\%) (MINAM 2009, 2015). Los sistemas agroforestales pueden reducir la deforestación de bosques naturales mediante un adecuado uso de los recursos naturales y una mayor productividad. En la actualidad los SAFs se desarrolla en el Perú principalmente en la región Amazónica. Donde se extendió con éxito esta práctica, los que en gran parte están diseñados a partir de la combinación de cultivos como el cacao, el café, entre otros frutales, con una vegetación arbórea de mediano y alto porte, la cual brinda sombra al cultivo y mejora su producción, otorgando principalmente al agricultor madera.

\section{Captura de carbono}

Los SAFs han llamado una especial atención por la captura de carbono que realizan debido a las ventajas percibidas por el gran volumen de biomasa aérea y los sistemas de raíces profundas que caracterizan a los árboles, que componen estos sistemas. Entonces en general, la vegetación arbórea o arbustiva ubicada dentro de los SAFs incrementa la captura de carbono considerablemente, debido principalmente al mayor volumen de su biomasa (Nair 1993). Por otro lado, es importante también notar que, al almacenar cantidades importantes de carbono en la biomasa aérea, a la par se pueden almacenar cantidades aún mayores de carbono en la biomasa subterránea (Nair et al. 2009). Estas cantidades de carbono almacenado en la biomasa principalmente van a depender de la proporción y tamaño de los árboles y arbustos (Montagnini y Nair 2004). La producción de biomasa aérea en diferentes tipos de SAFs y regiones ecológicas varía de $2,3 \mathrm{Mg} / \mathrm{ha}$ /año a $48 \mathrm{Mg} / \mathrm{ha} / \mathrm{año}$, dependiendo esta biomasa del número de componentes, estratos y arreglos espaciales y temporales (Young 1997).

La importancia de los SAFs en la captura de carbono se resume en dos razones: la primera es que el componente arbóreo captura el carbono atmosférico mediante la fotosíntesis, debido a que estos árboles son plantas perenes y lo almacenan, comportándose como activos sumideros de carbono por largos periodos de tiempo hasta que sean cortados o mueran. La segunda razón se debe a que los sistemas agroforestales reducen la necesidad de deforestar nuevos bosques tropicales y templados para la agricultura migratoria (Nair y Fernandes 1984, Winterbottom y Hazlewood 1987, Sanchez et al. 1990, Wiersum 1990, Pandey 2002). De acuerdo con Oelbermann et al. (2004), el potencial biológico de secuestro anual de carbono en biomasa aérea en SAFs de zonas tropicales es de $2,1 \times 109 \mathrm{Mg} / \mathrm{ha} / \mathrm{año}$, mientras en zonas templadas es de $1,9 \times 109 \mathrm{Mg} / \mathrm{ha} / \mathrm{año}$. Entonces se considera a los SAFs como sumideros de carbono (a pesar de que no fueron pensados originalmente para esta función), fuente de ingresos económicos adicionales al sistema y además podrían representar una alternativa aún más atractiva para el agricultor, considerando el pago de bonos de carbono de \$ 24.75 dólares americanos por $\mathrm{Mg} / \mathrm{ha} / \mathrm{año}$, precio promedio referido para el año 2020 en Sendeco2 (2021). Este dinero aumentará los ingresos obtenidos por la venta de los productos del cultivo agrícola y ayudará en el tiempo de espera hasta el crecimiento de las especies madereras, de las que se obtendrán ganancias solo después de muchos años de crecimiento para su posterior corte y venta como madera. Además, otro punto a resaltar sobre el uso de SAFs en el Perú es que las zonas tropicales tienen un mayor potencial en el secuestro de carbono que otras zonas como las templadas, áridas y semiáridas (Nair et al. 2009).

\section{Importancia de los sistemas agroforesta- les en el secuestro de carbono en el Perú}

$\mathrm{El} \mathrm{CO}_{2}$ es el gas con mayor importancia para el calentamiento global debido al inmenso volumen producido anualmente, el cual repres- 


\begin{tabular}{|c|c|c|c|c|c|c|c|c|c|c|c|c|}
\hline $\begin{array}{l}\text { 喪 } \\
\text { 至 } \\
\text { 点 }\end{array}$ & \multicolumn{5}{|c|}{ 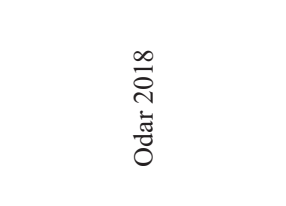 } & 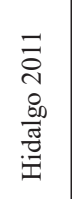 & \multicolumn{3}{|c|}{ 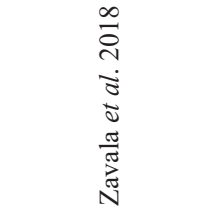 } & \multicolumn{3}{|c|}{ 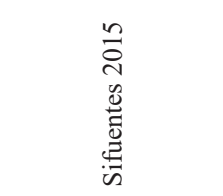 } \\
\hline $\begin{array}{l}\text { EDAD DEL CULTIVO } \\
\text { (AÑOS) }\end{array}$ & ' & 1 & & ' & ' & ' & 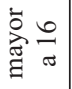 & $\begin{array}{ll}\infty & 0 \\
0 & 0 \\
0 & 0 \\
0 & >\end{array}$ & $\begin{array}{ll}0 & \infty \\
\overline{0} & 8 \\
\ddot{g} & 8\end{array}$ & & ' & ' \\
\hline $\begin{array}{l}\text { FLUJO ANUAL DE C } \\
\text { (Mg/ha/año) }\end{array}$ & ' & 1 & ' & ' & ' & ' & ' & ' & ' & $\begin{array}{l}\hat{y} \\
y \\
y\end{array}$ & $\hat{a}$ & $\begin{array}{l}\text { ?o } \\
\text { in } \\
\text { in }\end{array}$ \\
\hline $\begin{array}{l}\text { CARBONO TOTAL } \\
(\mathrm{Mg} / \mathrm{ha})\end{array}$ & ' & 1 & ' & ' & ' & $\begin{array}{l}\stackrel{i}{r} \\
\stackrel{n}{n}\end{array}$ & 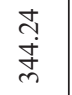 & $\begin{array}{l}\vec{b} \\
\infty \\
\stackrel{\infty}{=}\end{array}$ & $\begin{array}{l}\vec{\sigma} \\
\dot{H}\end{array}$ & & ' & ' \\
\hline $\begin{array}{c}\text { CARBONO DEL SUELO } \\
(\mathrm{Mg} / \mathrm{ha})\end{array}$ & ' & ' & & ' & ' & $\stackrel{\infty}{\stackrel{\infty}{\beth}}$ & $\begin{array}{l}\infty \\
\stackrel{0}{1} \\
\text { in }\end{array}$ & 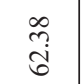 & $\frac{6}{8}$ & & ' & ' \\
\hline $\begin{array}{c}\text { CARBONO AEREO } \\
\text { (Mg/ha) }\end{array}$ & 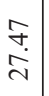 & $\begin{array}{l}\stackrel{8}{0} \\
\dot{0} \\
\text { iे }\end{array}$ & $\begin{array}{l}\tilde{D} \\
0 \\
\\
\end{array}$ & \begin{tabular}{l|l}
0 & $\bar{\infty}$ \\
$\infty$ & $\infty$ \\
\hdashline & $\infty$ \\
$=$ & 0
\end{tabular} & 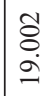 & $\begin{array}{l}\infty \\
\stackrel{m}{a}\end{array}$ & $\begin{array}{l}0 \\
\stackrel{0}{10} \\
\infty \\
\sim\end{array}$ & 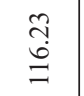 & $\begin{array}{l}n \\
\infty \\
\infty \\
\infty\end{array}$ & $\begin{array}{l}\mathfrak{b} \\
\mathfrak{i} \\
=\end{array}$ & $\stackrel{\infty}{\stackrel{\infty}{n}}$ & $\begin{array}{l}\vec{a} \\
\text { à }\end{array}$ \\
\hline 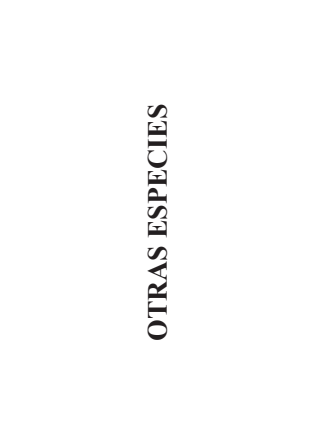 & & 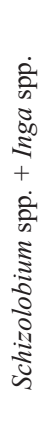 & & 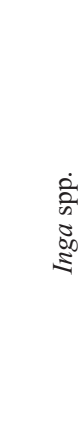 & & 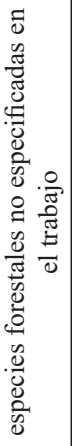 & & 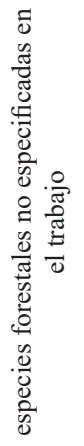 & & 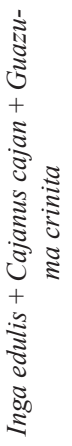 & 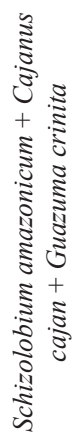 & 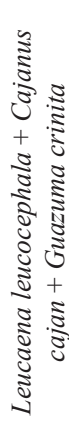 \\
\hline 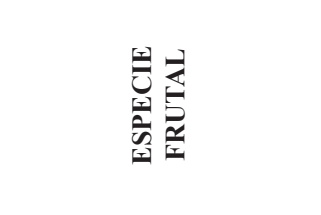 & & & 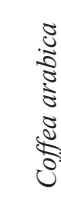 & & & 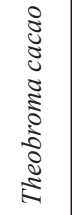 & & 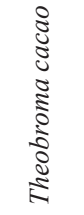 & & & 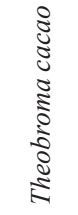 & \\
\hline$\widehat{\mathrm{Z}}$ & & & 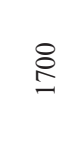 & & & 8 & & $\stackrel{n}{\stackrel{n}{r}}$ & & & : & \\
\hline 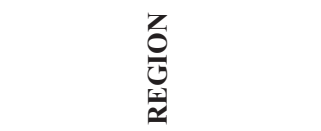 & & & 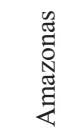 & & & 芞 & & 总 & & & 总 & \\
\hline
\end{tabular}

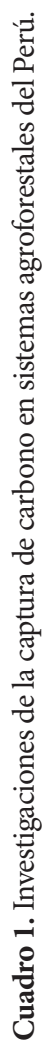




\begin{tabular}{|c|c|c|c|c|c|c|c|c|c|c|c|c|c|c|c|c|c|c|c|}
\hline 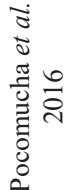 & \multicolumn{6}{|c|}{ 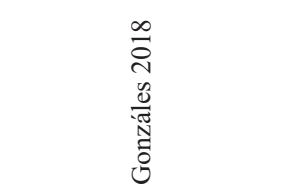 } & \multicolumn{2}{|c|}{ 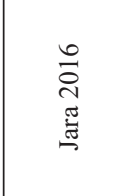 } & \multicolumn{3}{|c|}{ 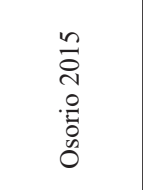 } & \multicolumn{6}{|c|}{ 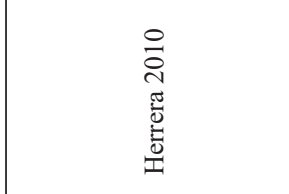 } & \multicolumn{2}{|c|}{ 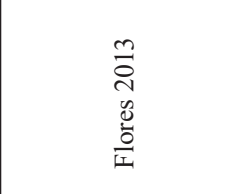 } \\
\hline ' & $\nabla$ & $r$ & $\nabla$ & r & + & $r$ & $n$ & 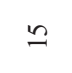 & in & $n$ & in & 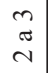 & $\begin{array}{c}J \\
\sigma \\
m\end{array}$ & $\begin{array}{l}n \\
\tilde{\sigma} \\
f \\
f\end{array}$ & $\begin{array}{l}n \\
\tilde{n} \\
\sim\end{array} \mid$ & $\begin{array}{c}+ \\
\pi \\
m\end{array}$ & $\begin{array}{l}n \\
\infty \\
+\end{array}$ & ' & ' \\
\hline ' & , & , & I & ' & , & I & ' & ' & ' & ' & ' & 1 & 1 & . & ' & ' & I & \begin{tabular}{l|l}
1 & 1
\end{tabular} & ' \\
\hline$\stackrel{\infty}{\stackrel{\infty}{m}}$ & $\begin{array}{l}\stackrel{\Xi}{\sim} \\
\underset{\mathbb{I}}{\mathbb{N}}\end{array}$ & $\begin{array}{c}\stackrel{1}{\infty} \\
\infty \\
\dot{D} \\
\dot{\infty}\end{array}$ & $\begin{array}{l} \pm \\
\stackrel{\sim}{2}\end{array}$ & 足 & 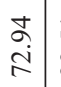 & $\begin{array}{l}\bar{n} \\
2\end{array}$ & ' & ' & $\infty$ & $\begin{array}{l}\vec{n} \\
\pm \\
\Xi\end{array}$ & $\begin{array}{l}\stackrel{0}{2} \\
\stackrel{a}{g} \\
\end{array}$ & ले & $\begin{array}{l}n \\
\stackrel{n}{f} \\
\dot{p}\end{array}$ & $\underset{\bar{\sigma}}{=}$ & $\begin{array}{l}2 \\
\dot{m} \\
\dot{m}\end{array} \mid$ & $\begin{array}{l}1 \\
\dot{0} \\
\dot{q}\end{array}$ & $\underset{\dot{i}}{\vec{i}}$ & ' & ' \\
\hline $\begin{array}{l}n \\
n \\
b\end{array}$ & $\begin{array}{l}\text { ஃे } \\
\text { கె் }\end{array}$ & $\begin{array}{l}\stackrel{n}{\hat{i}} \\
\stackrel{i}{i}\end{array}$ & $\begin{array}{l}\hat{\sigma} \\
\stackrel{0}{\circ}\end{array}$ & $\mid \begin{array}{l}\hat{0} \\
\infty \\
\infty \\
\infty\end{array}$ & $\vec{\forall}$ & 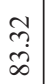 & ' & ' & $\stackrel{m}{m}$ & 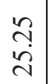 & $\begin{array}{l}\vec{b} \\
i \\
m\end{array}$ & $\underset{\dot{J}}{\vec{\Xi}}$ & $\stackrel{n}{\stackrel{2}{r}}$ & $\begin{array}{l}9 \\
\infty \\
\infty\end{array}$ & 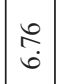 & $\begin{array}{l}\infty \\
n \\
\infty \\
\infty\end{array}$ & $\vec{a}$ & $\begin{array}{l}1 \\
\end{array}$ & ' \\
\hline 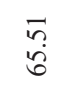 & $\begin{array}{c}\infty \\
\stackrel{\Xi}{\Xi} \\
\Xi\end{array}$ & 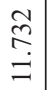 & $\stackrel{\sim}{a}$ & 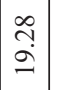 & $\underset{m}{\stackrel{+}{-}}$ & $\stackrel{9}{6}$ & $\begin{array}{l}\stackrel{+}{+} \\
\dot{J}\end{array}$ & 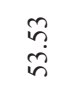 & $\begin{array}{l}0 \\
i \\
\text { i }\end{array}$ & $\begin{array}{l}\tilde{n} \\
\tilde{\infty} \\
\infty\end{array}$ & 市 & $\begin{array}{l}0 \\
\stackrel{\sim}{\sim}\end{array}$ & $\begin{array}{l}2 \\
\grave{n} \\
\tilde{m}\end{array}$ & 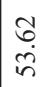 & 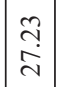 & $\begin{array}{l}\underset{i}{d} \\
\stackrel{m}{ }\end{array}$ & $\overrightarrow{\stackrel{f}{f}}$ & 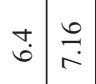 & $\stackrel{\circ}{\stackrel{0}{0}}$ \\
\hline 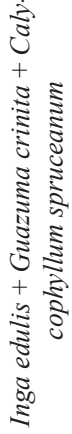 & & & $\begin{array}{l}\dot{0} \\
\text { के } \\
0 \\
0 \\
\Xi\end{array}$ & है: & & & 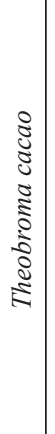 & 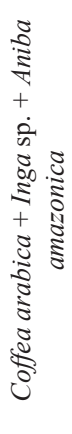 & & 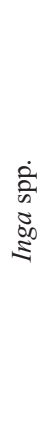 & & & 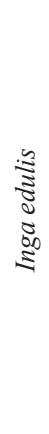 & & & 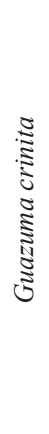 & & 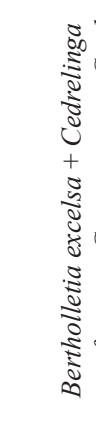 & 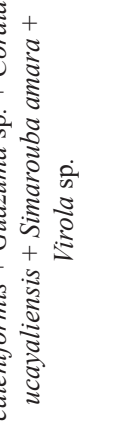 \\
\hline 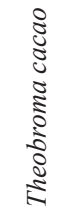 & & & 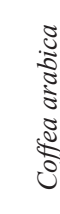 & לֶ. & & & & 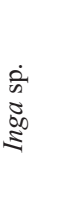 & & 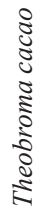 & & & & 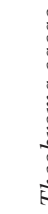 & 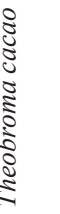 & & & 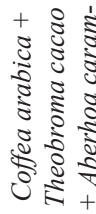 & 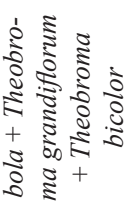 \\
\hline \& & 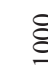 & & $\underset{\infty}{\stackrel{\infty}{-}}$ & 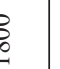 & \& & & & \&ू & $\vec{\sim}$ & $\stackrel{\infty}{\infty}$ & $\stackrel{\cong}{\Xi}$ & & & $S$ & 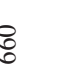 & & & & ஓ \\
\hline 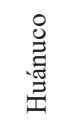 & & & 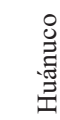 & : & & & & 蒿 & & 兽 & & & & & 送 & & & & \\
\hline
\end{tabular}




\begin{tabular}{|c|c|c|c|c|c|c|c|c|c|}
\hline 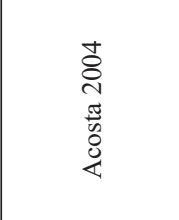 & & 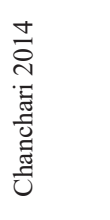 & 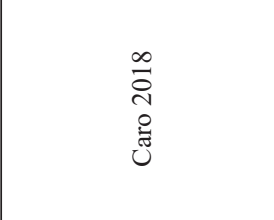 & \multicolumn{4}{|c|}{ 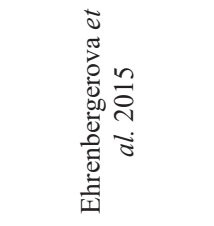 } & \multicolumn{2}{|c|}{ 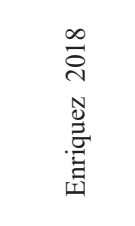 } \\
\hline ' & $\nabla$ & 0 & ' & $r$ & $\cong$ & 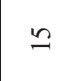 & ' & 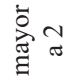 & 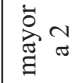 \\
\hline ' & ' & ' & . & ' & , & ' & ' & ' & ' \\
\hline $\begin{array}{l}\infty \\
\infty \\
\infty \\
\infty \\
\infty\end{array}$ & ' & ' & ' & $\begin{array}{l}n \\
\tilde{n} \\
\underline{-}\end{array}$ & $\stackrel{\partial}{\partial}$ & $\stackrel{n}{\Sigma}$ & $\hat{\sigma}$ & ' & ' \\
\hline$\hat{\hat{j}}$ & ' & ' & ' & \begin{tabular}{|l|}
$\infty$ \\
$\infty$ \\
0 \\
0
\end{tabular} & $\begin{array}{l}0 \\
\dot{\infty}\end{array}$ & $\stackrel{+}{\stackrel{\leftrightarrow}{=}}$ & $\stackrel{\wedge}{\infty}$ & $\begin{array}{l}0 \\
\infty \\
\stackrel{0}{0}\end{array}$ & $\stackrel{m}{\infty}$ \\
\hline $\begin{array}{l}\overrightarrow{0} \\
\stackrel{0}{0} \\
\text { N }\end{array}$ & $\begin{array}{l}\stackrel{0}{2} \\
\stackrel{ \pm}{ \pm}\end{array}$ & $\vec{\infty}$ & ণ̃ & $n$ & $\stackrel{m}{\stackrel{n}{m}}$ & $\widehat{\sigma}$ & - & ' & ' \\
\hline 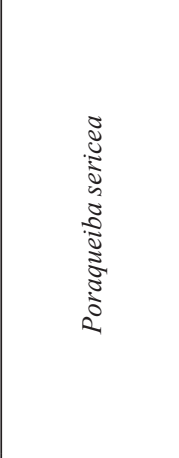 & . & 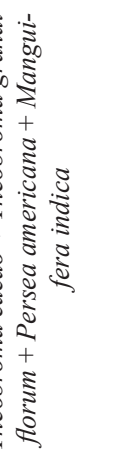 & 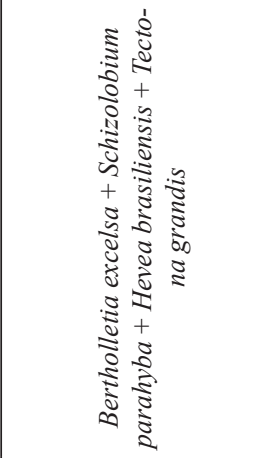 & 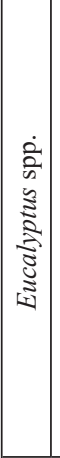 & 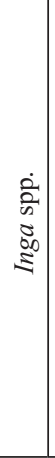 & 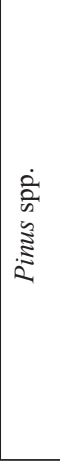 & ' & & 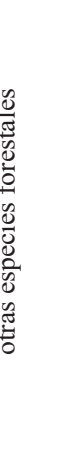 \\
\hline 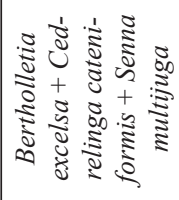 & $\mid \begin{array}{l}0 \\
0 \\
0 \\
0 \\
0 \\
0 \\
0 \\
0 \\
0 \\
0 \\
0 \\
0\end{array}$ & 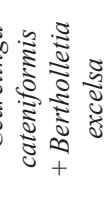 & 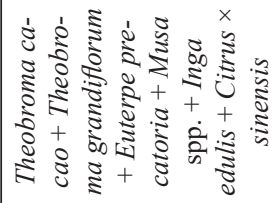 & & & 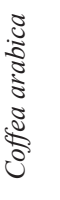 & & 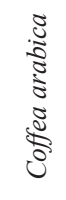 & 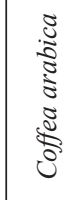 \\
\hline 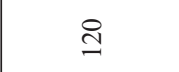 & $\Xi$ & $\stackrel{\nabla}{=}$ & 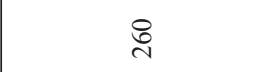 & $\begin{array}{l}8 \\
8 \\
0\end{array}$ & $\begin{array}{l}8 \\
n \\
n \\
-1\end{array}$ & 운 & $\begin{array}{l}0 \\
n \\
n\end{array}$ & & $\frac{n}{f}$ \\
\hline 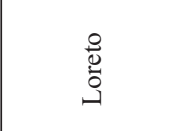 & & $\begin{array}{l}\stackrel{8}{0} \\
0 \\
0 \\
\end{array}$ & 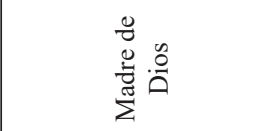 & & & $\begin{array}{l}8 \\
0 \\
0 \\
0 \\
0\end{array}$ & & & छ \\
\hline
\end{tabular}




\begin{tabular}{|c|c|c|c|c|c|c|c|c|c|c|c|}
\hline & & & 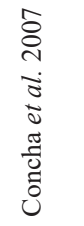 & & & $\begin{array}{l}\stackrel{J}{\circ} \\
\text { N } \\
\text { 峁 } \\
\frac{0}{5}\end{array}$ & & 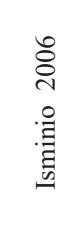 & & & 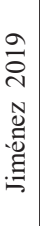 \\
\hline ิ & ิ & $n$ & $\simeq$ & $\simeq$ & in & $\simeq$ & $m$ & + & $n$ & 0 & + \\
\hline$\dddot{n}$ & $\underset{\infty}{m}$ & $\vec{\sim}$ & $\ddot{\vartheta}$ & $\ddot{\ddot{g}}$ & $\stackrel{\sim}{2}$ & ' & 1 & ' & 1 & ' & ' \\
\hline ' & ' & 1 & ' & ' & ' & ' & $\begin{array}{l}\hat{\sigma} \\
\stackrel{+}{ \pm} \\
\stackrel{J}{*}\end{array}$ & $\begin{array}{l} \pm \\
\vec{\sigma} \\
\dot{0}\end{array}$ & $\begin{array}{l}\infty \\
\stackrel{\sim}{\vartheta} \\
\stackrel{\vec{v}}{\sim}\end{array}$ & $\begin{array}{l}\stackrel{+}{\leftrightarrows} \\
\underset{\vec{j}}{m}\end{array}$ & $\begin{array}{l}\vec{n} \\
\\
\end{array}$ \\
\hline ' & ' & 1 & ' & ' & ' & ' & $\begin{array}{l}\text { శે } \\
\text { } \\
\text { in }\end{array}$ & $\underset{m}{\stackrel{m}{m}}$ & $\begin{array}{l}\tilde{\sigma} \\
\text { in }\end{array}$ & $\frac{a}{\stackrel{g}{a}}$ & 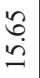 \\
\hline$\hat{\hat{q}}$ & $\begin{array}{l}\infty \\
\infty \\
\dot{f}\end{array}$ & 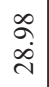 & $\begin{array}{l}\infty \\
\stackrel{\circ}{+} \\
\dot{f}\end{array}$ & $\begin{array}{l}\hat{\sigma} \\
\dot{y}\end{array}$ & $\begin{array}{l}\text { స్ } \\
\text { ֶె. }\end{array}$ & $\begin{array}{l}\check{\hat{i}} \\
\tilde{\lambda}\end{array}$ & 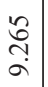 & $\begin{array}{l}\vec{b} \\
\stackrel{n}{a} \\
\end{array}$ & $\begin{array}{l}n \\
n \\
n \\
n\end{array}$ & 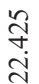 & $\begin{array}{l}\infty \\
\stackrel{0}{\circ}\end{array}$ \\
\hline 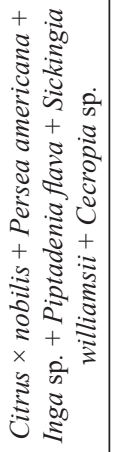 & 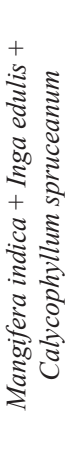 & $\begin{array}{l}\dot{0} \\
\dot{2} \\
8 \\
\Xi \\
\Xi\end{array}$ & 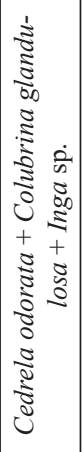 & 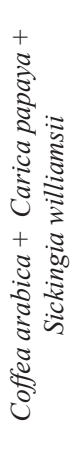 & 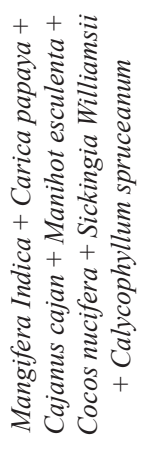 & 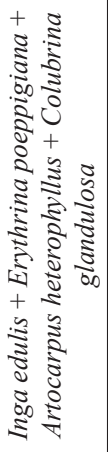 & & 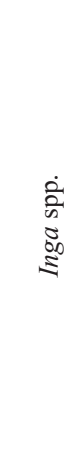 & & & 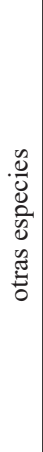 \\
\hline & & & 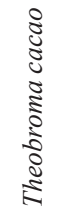 & & & 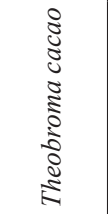 & & 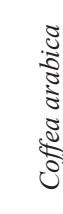 & & & 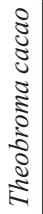 \\
\hline$\stackrel{\infty}{+}$ & ஸે & $\begin{array}{l}0 \\
\stackrel{2}{2}\end{array}$ & in & ते & $\stackrel{\text { ণे }}{2}$ & ले & & $\stackrel{ }{1}$ & & & $\hat{m}$ \\
\hline & & & 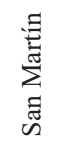 & & & 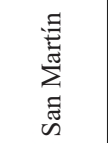 & & 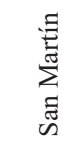 & & & 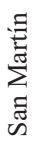 \\
\hline
\end{tabular}




\begin{tabular}{|c|c|c|c|c|c|c|c|c|c|c|}
\hline & & & & & \multicolumn{5}{|c|}{ 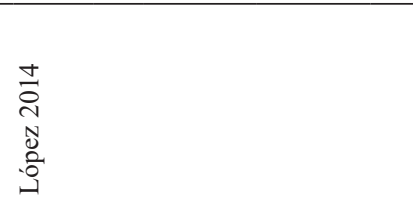 } & \multirow{2}{*}{ 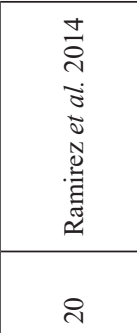 } \\
\hline ' & ' & ' & 1 & ' & ' & 1 & ' & ' & ' & \\
\hline ' & ' & ' & 1 & ' & ' & 1 & ' & ' & ' & $\stackrel{?}{f}$ \\
\hline ' & ' & ' & ' & ' & ' & ' & ' & ' & ' & $\exists$ \\
\hline ' & ' & ' & ' & ' & ' & 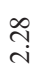 & ' & ' & ' & $\begin{array}{l}b \\
\ddot{a}\end{array}$ \\
\hline $\begin{array}{l}\infty \\
\infty \\
\infty \\
\infty\end{array}$ & ले & $\stackrel{\mathbb{N}}{\stackrel{i}{i}}$ & $\begin{array}{l}\infty \\
\stackrel{0}{0} \\
\stackrel{0}{0}\end{array}$ & $\stackrel{尺}{\stackrel{2}{r}}$ & ఫ̆ & ' & $\stackrel{\text { fq }}{=}$ & $\begin{array}{l}\vec{n} \\
i n\end{array}$ & $\begin{array}{l}\hat{\infty} \\
\dot{\nabla}\end{array}$ & $\begin{array}{l}n \\
\infty \\
\infty\end{array}$ \\
\hline 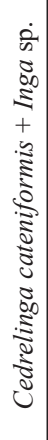 & 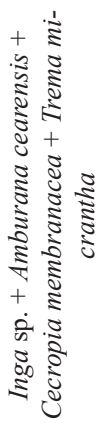 & 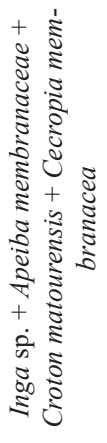 & 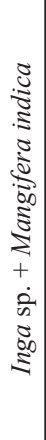 & 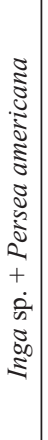 & 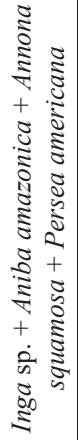 & ' & 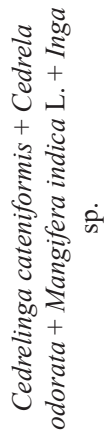 & 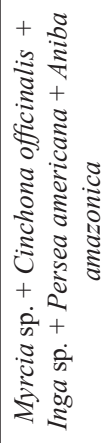 & 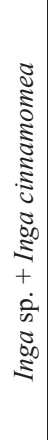 & 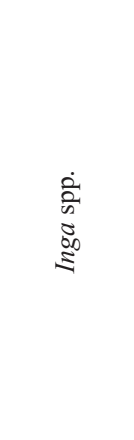 \\
\hline & & & & & 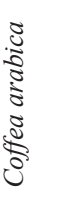 & & & & & 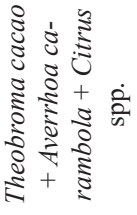 \\
\hline & & & & & & & 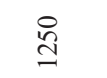 & & & $\stackrel{\stackrel{+}{n}}{=}$ \\
\hline & & & & & $\sum_{\Xi}^{\Xi}$ & & & & & 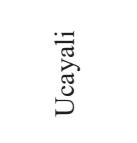 \\
\hline
\end{tabular}




\begin{tabular}{|c|c|c|c|c|c|c|c|c|c|}
\hline \multicolumn{10}{|c|}{ 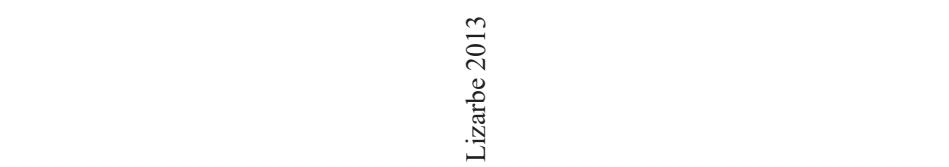 } \\
\hline \multirow[t]{2}{*}{$\infty$} & in & $m$ & $\begin{array}{l}\infty \\
\stackrel{0}{\Xi} \\
\stackrel{\Xi}{d}\end{array}$ & $\sigma$ & $r$ & 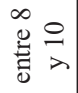 & $\infty$ & 으 & 으 \\
\hline & ' & ' & I & ' & ' & ' & ' & ' & I \\
\hline ' & ' & , & 1 & I & I & I & , & ' & ' \\
\hline ' & I & , & ' & I & I & I & I & ' & I \\
\hline $\begin{array}{l}\hat{\infty} \\
\stackrel{\Xi}{\beth}\end{array}$ & 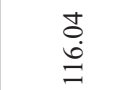 & $\begin{array}{l}d \\
\dot{d} \\
=\end{array}$ & \begin{tabular}{l}
$\infty$ \\
\multirow{1}{0}{} \\
$\infty$ \\
0
\end{tabular} & $\stackrel{\sim}{\stackrel{2}{a}}$ & 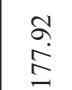 & $\frac{1}{\stackrel{5}{a}}$ & $\begin{array}{l}\infty \\
n \\
\infty \\
\infty\end{array}$ & $\stackrel{m}{m}$ & 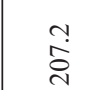 \\
\hline 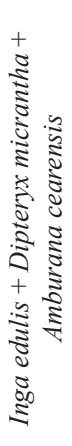 & 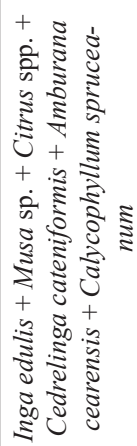 & 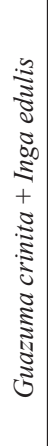 & 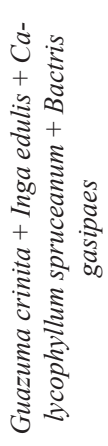 & 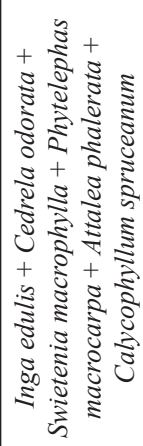 & 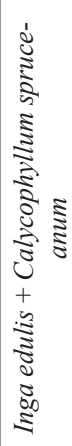 & 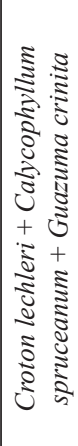 & 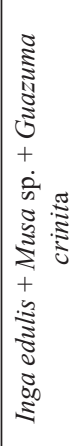 & 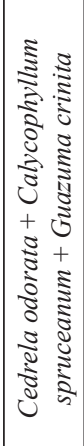 & 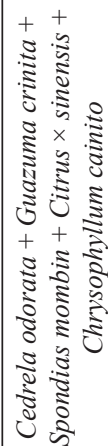 \\
\hline & & & & 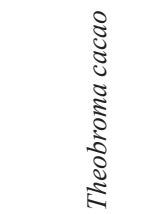 & & & & & \\
\hline & & & & సิ & & & & & \\
\hline & & & & 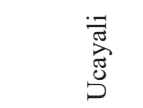 & & & & & \\
\hline
\end{tabular}


senta el 50\% de los gases de efecto invernadero (Jobbágy y Jackson 2000). El secuestro de carbono consiste en la captura del $\mathrm{CO}_{2}$ del ambiente y su almacenamiento en depósitos de larga vida, como la biomasa vegetal aérea (estructuras como el tronco, hojas, flores y frutos) y la biomasa subterránea (raíces, microorganismos y las formas estables de carbono orgánico e inorgánico en el suelo) (Palm et al. 2004, Haile et al. 2008). Los bosques son los ecosistemas terrestres que almacenan la mayor cantidad de carbono tanto en su vegetación como debajo del suelo, representando un papel importante en el intercambio de $\mathrm{CO}_{2}$ entre la biosfera y la atmosfera (Jaramillo 2004).

La característica de los SAFs de incluir árboles en su composición hace que sean más similares a los bosques. Por esta razón se cree que los SAFs tienen un mayor potencial para el secuestro de carbono tanto aéreo como subterráneo, que otras formas de uso de la tierra como son los monocultivos o los pastizales (Sanchez 2000, Roshetko et al. 2002, Kirby y Potvin 2007). La agroforestería fomenta prácticas sostenibles con bajos insumos que logran minimizar la alteración de los suelos y las plantas, resaltando por la vegetación perenne y el reciclaje de nutrientes, que contribuyen al almacenamiento de carbono a largo plazo (Nair 2004). A pesar, del hecho de que los SAFs no fueron diseñados principalmente para la captura de carbono, brindan la oportunidad de aumentar las reservas de carbono en la biosfera terrestre (Casanova-Lugo et al. 2011, Petit-Aldana et al. 2012).

El Perú tiene un enorme potencial para el desarrollo en el sector forestal, debido al importante componente de carbono negociable en el mercado internacional. Las áreas de los SAFs están en aumento en el Perú, debido a sus múltiples beneficios tanto ecológicos como económicos; con especies forestales principalmente de interés maderero como cedro (Cedrela odorata L.), caoba (Swietenia macrophylla King), pashaco (Schizolobium spp.), shihuahuaco (Dipteryx micrantha Harms), tornillo (Cedrelinga cateniformis (Ducke) Ducke), bolaina (Guazuma crinita Mart.), capirona (Caly- cophyllum spruceanum (Benth.) Hook. f. ex K. Schum.), pino (Pinus sp.) y eucalipto (Eucalyptus sp.); especies frutales como: guaba (Inga edulis Mart.), plátano (Musa sp.), mandarina (Citrus $\times$ nobilis Lour.), limón (Citrus sp.), pijuayo (Bactris gasipaes Kunth), caimito (Chrysophyllum cainito L.), castaña (Bertholletia excelsa Bonpl.), mango (Mangifera indica L.) y palta (Persea americana Mill.); que protegen del sol principalmente a cultivos como: café (Coffea arabica L.), cacao (Theobroma cacao L.) y copazú (Theobroma grandiflorum (Willd. ex Spreng.) K. Schum.), que representan los principales productos de exportación en el Perú (Isminio 2006, Concha et al. 2007, Herrera 2010, Hidalgo 2011, Lizarbe 2013, López 2014, Ehrenbergerova et al. 2015, Gonzáles 2018, Zavala et al. 2018, Jiménez 2019)

En esta investigación realizamos la revisión de estudios de captura de carbono realizados en sistemas agroforestales del Perú y analizamos los resultados de los diferentes sistemas agroforestales y observamos la edad, área y especies (forestales y frutales) de cada sistema agroforestal; así como, el carbono almacenado en el suelo y la parte aérea y el total de cada sistema agroforestal. Actualmente existen pocos estudios de cuantificación del secuestro de carbono en los sistemas agroforestales peruanos tanto de la biomasa aérea como del suelo. Este estudio analizó 21 investigaciones de la captura de carbono (Cuadro 1) principalmente en la Amazonía peruana tanto en selva alta como en selva baja, que muestran la variación de captura de carbono de acuerdo con la composición de cada SAF. Entre las regiones que cuentan con estudios de captura de carbono en sistemas agroforestales en el Perú tenemos a Huánuco, San Martin, Ucayali, Loreto, Pasco, Amazonas, Madre de Dios y Puno. La mayoría de los estudios son tesis tanto de pregrado como de posgrado que evalúan sistemas agroforestales con cultivos de café (Coffea arabica) o cacao (Theobroma cacao) entre las especies frutales asociados con variadas especies forestales maderables de alta calidad como el cedro (Cedrela odorata), shihuanhuaco (Dipteryx micrantha) y tornillo (Cedrelinga cateniformis). 
En la Cuadro 1 podemos observar que Zavala et al. (2018) encontraron el mayor sumidero de carbono en SAFs de cacao (Theobroma cacao) y otras especies forestales en la región Huánuco en una parcela de más de 16 años que tiene un stock de carbono sobre el suelo de $285,16 \mathrm{Mg} /$ ha, bajo el suelo de 59,08 Mg/ha y un total de $344,24 \mathrm{Mg} / \mathrm{ha}$. En segundo lugar, está el SAF de castaña (Bertholletia excelsa), umari (Poraqueiba sericea Tul.), tornillo (Cedrelinga cateniformis) y pashaco (Senna multijuga (Rich.) H.S. Irwin \& Barneby) de edad no descrita por Acosta (2004) en la región Loreto con un stock de carbono sobre el suelo de $265,01 \mathrm{Mg} /$ ha, bajo el suelo de $23,97 \mathrm{Mg} / \mathrm{ha}$ y un total de $288,98 \mathrm{Mg} / \mathrm{ha}$. También podemos observar que en la investigación de Lizarbe (2013) entre los SAFs de cacao (Theobroma cacao) y otras especies forestales de diferentes edades entre 3 y 10 años en la región Ucayali, tienen un rango de 207,2 a $73,33 \mathrm{Mg} / \mathrm{ha}$ de stock de carbono sobre el suelo. Sin embargo, es difícil comparar el stock de carbono de estos SAFs porque tienen diferentes edades y el carbono se acumula con el tiempo, por lo que es mejor y es más comparable analizar el flujo de carbono o captura de carbono por año.

Entre las principales limitaciones de los estudios de captura de carbono en SAFs en el Perú se tiene la falta de estandarización de la metodología. Además, la confusión de los términos de biomasa y cantidad de carbono; captura de carbono y stock de carbono. También, observamos que pocos estudios son rigurosos con todos los parámetros de evaluación como son: biomasa vegetal (cultivo, árboles y arbustos), hojarasca, necromasa (árboles muertos en pie y árboles muertos caídos), carbono de las raíces y carbono en el suelo. También, la falta de información sobre la estructura de los sistemas agroforestales (especies, edad de la plantación, densidad de árboles) y de las características del área de estudio (altitud, clima, ubicación geográfica) producen estudios poco rigurosos e incapaces de hacer comparaciones y obtener resultados a gran escala.

Con el calentamiento global que pone en riesgo nuestro futuro y el crecimiento de la po- blación mundial, una alternativa sostenible de uso de tierra son los sistemas agroforestales, estos están generando interés no solo en el mundo académico sino también en el público en general debido a que puede representar ganancias económicas para el sector agropecuario. Es necesario realizar mayores investigaciones para evaluar con más precisión el potencial de los SAFs en los trópicos, donde los SAFs son extensivamente practicados presentando un alto potencial, lo que servirá para ayudar a dilucidar los procesos elementales que impulsan o limitan el uso de estos sistemas. El Perú posee una geografía privilegiada dentro del neotrópico, con una amplia gradiente altitudinal desde los andes hasta la amazonia, que permite el diseño de diferentes SAFs con innumerables combinaciones de especies adecuadas para cada piso altitudinal y que logren beneficiar el sector económico de la agricultura y la ganadería peruana. Estas investigaciones mejoraran las prácticas para que sean más sostenibles tanto económica como ecológicamente.

\section{Conclusiones}

La captura de carbono en los sistemas agroforestales en el Perú ha sido estudiado en muchos regiones de la amazonia, donde sobresalen los sistemas agroforestales de Huánuco y Loreto, que fueron los que tienen más potencial en el almacenamiento de carbono, el primero con un stock de carbono de 344,24 Mg/ha en un sistema agroforestal de cacao (Theobroma cacao) y otras especies forestales de más de 16 años y el segundo con 288,98 Mg/ha en un sistema agroforestal de castaña (Bertholletia excelsa), umari (Poraqueiba sericea), tornillo (Cedrelinga cateniformis) y pashaco (Senna multijuga) sin edad conocida. Esto representa un gran potencial en el secuestro de carbono por los sistemas agroforestales en el Perú, un país tropical y con un territorio mayormente amazónico. Todas estas ventajas podrían favorecer la instalación de nuevos sistemas agroforestales en tierras degradadas por la agricultura migratoria y así evitar más deforestación dentro de un país megadiverso. Por otro lado, se encontró una falta de estudios de captura de 
carbono, que brindaría datos de la captura de carbono de los sistemas agroforestales en un intervalo de tiempo, esto ayudaría mucho en el análisis de datos de los sistemas agroforestales, ya que podrían ser comparables entre diferentes sistemas agroforestales en todo el Perú.

Así también; se requieren de más estudios en la conservación de suelos, agua y biodiversidad para mejorar esta tecnología y convertirla en la mejor solución a la deforestación y cambio de uso de suelos. La mayoría de los estudios realizados en el Perú en este tema fueron realizados en pocas regiones y por investigadores de forma personal por motivo de tesis, lo cual muestra que es poco el interés por las instituciones gubernamentales, quienes deberían fomentar la investigación y practica de los sistemas agroforestales en el Perú.

\section{Referencias}

Acosta, JE. 2004. Determinación de captura de carbono en dos tipos de bosque con manejo (sistema agroforestal) y sin manejo (bosque secundario) en la localidad de Zungarococha (Iquitos) (en línea). Tesis Ing. Iquitos, Perú, UNAP. 107 p. Consultado 21 oct. 2020. Disponible en http://repositorio.unapiquitos.edu. pe/handle/UNAP/4726

Allen, SC; Shibu, J; Nair, PKR; Brecke, BJ; Nkedi-Kizza, P; Ramsey, CL. 2004. Safety-net role of tree roots: experimental evidence from an pecan (Carya illinoensis K. Koch)-cotton (Gossypium hirsutum L.) alley cropping system in the southern United States. Forest Ecology and Management 192(2-3):395-407. DOI: https:// doi.org/10.1016/j.foreco.2004.02.009.

Beer, J; Harvey, C; Ibrahim, M; Harmand, JM; Somarriba, E; Jiménez, F. 2003. Servicios ambientales de los sistemas agroforestales. Agroforestería en Las Américas 10(37-38):80-87. DOI: https://doi.org/10.5154/r.rchscfa.2015.06.029.

Caro, DM. 2018. Secuestro de carbono en sistemas agroforestales de los distritos de las Piedras y Tambopata, provincia Tambopata, departamento de Madre de Dios-Perú (en línea). Madre de Dios, Perú, UNAMAD. Consultado
21 oct. 2020. Disponible en http://repositorio. unamad.edu.pe/handle/UNAMAD/520.

Casanova-Lugo, F; Petit-Aldana, J; Solorio-Sánchez, J. 2011. Los sistemas agroforestales como alternativa a la captura de carbono en el trópico mexicano. Revista Chapingo (Serie ciencias forestales y del ambiente) 17(1):133143. DOI: https://doi.org/10.5154/r.rchscfa.2010.08.047.

Casanova-Lugo, F; Ramirez-Aviles, L; David, P; Caamal-Maldonado, A; Piñeiro-Vázquez, AT; Díaz-Echeverria, V. 2016. Enviromental services from tropical agroforestry systems. Revista Chapingo (Serie ciencias forestales y del ambiente) 22(3):269-284.

Chanchari, EM 2014. Almacenamiento de carbono en relacion con la topografia y edad de los sistemas agroforestales de la carretera Iquitos - Nauta, Perú, 2012 (en línea). Iquitos, Perú, Universidad Nacional de la Amazonia Peruana. Consultado 21 oct. 2020. Disponible en https://repositorio.unapiquitos.edu.pe/handle/20.500.12737/4309.

Concha, JY; Alegre, JC; Pocomucha, V. 2007. Determinacion de las reservas de carbono en la biomasa aérea de sistemas agroforestales de Theobroma cacao L. en el departamento de San Martin, Perú. Ecologia Aplicada 6(1-2):75-82.

Ehrenbergerova, L; Cienciala, E; Kučera, A; Guy, L; Habrová, H 2015. Carbon stock in agroforestry coffee plantations with different shade trees in Villa Rica, Peru. Agroforestry Systems 90(3):433-445. DOI: https://doi.org/10.1007/ s10457-015-9865-Z.

Enriquez, LM. 2018. Determinación de captura de carbono en suelos de un sistema agroforestal con café (Coffea arabica) en el valle Esquilaya-región Puno, 2018 (en línea). Tesis Ing. Lima, Perú, Universidad Peruana Unión. Consultado 21 oct. 2020. Disponible en https:// repositorio.upeu.edu.pe/handle/UPEU/1712.

Flores, SA 2013. Situación actual de la reforestacion con sistemas agroforestales para captura de carbono, en tres comunidades de la carretera Iquitos - Nauta, región Loreto (en línea). Tesis Ing. Iquitos, Perú, UNAP. Consultado 21 
oct. 2020. Disonible en https://1library.co/document/zlg48d2y-situacion-reforestacion-sistemas-agroforestales-comunidades-carretera-iquitos-region.html.

Garrett, HE; McGraw, RL; Walter, WD. 2009. Alley cropping practices. In Garrettt, HE (ed.). North American agroforestry: an integrated science and practice. 2 ed. Madison, Estados Unidos de América, ASA. p. 133-162. DOI: https://doi.org/10.2134/2009.northamericanagroforestry.2ed.c7.

Gold, MA; Garrett, HE. 2009. Agroforestry nomenclature, concepts, and practices. In Garrettt, HE (ed.). North American agroforestry: an integrated science and practice. 2 ed. Madison, Estados Unidos de América, ASA. p. 45-56. DOI: https://doi.org/10.2134/2009.northamericanagroforestry.2ed.c3.

Gonzáles, JF. 2018. Carbono almacenado en sistemas agroforestales de Coffea arabica L. "café" de 4 y 7 años en relación a la gradiente altitudinal, Huánuco (en línea). Huánuco, Perú, UNAS. Consultado 21 oct. 2020. Disponible en http://repositorio.unas.edu.pe/handle/ UNAS/1476.

Haile, SG; Nair, PKR; Nair, VD. 2008. Carbon storage of different soil-size fractions in Florida silvopastoral systems. Journal of Environmental Quality 37(5):1789-1797. DOI: https://doi. org/10.2134/jeq2007.0509.

Herrera, JB. 2010. Estimación de la biomasa y carbono almacenado en dos sistemas agroforestales de cacao (Theobroma cacao L.) de diferentes edades en la Provincia de Leoncio Prado (en línea). Huánuco, Perú, UNAS. Consultado 21 oct. 2020. Disponible en http://repositorio. unas.edu.pe/handle/UNAS/123.

Hidalgo, P. 2011. Determinación de las reservas totales de carbono en un sistema agroforestal de la Selva Alta de Tingo María. Aporte Santiaguino 4(1):87-92.

Isminio, MA. 2006. Estimación del carbono en la biomasa aérea del café (Coffea arabica var. catimor) bajo sombra de guaba (Inga edulis) en la provincia de Lamas-Perú (en línea). Tarapoto, Perú, UNSM. Consultado 21 de oct. 2020.
Disponible en http://repositorio.unsm.edu.pe/ handle/11458/795.

Jara, RA. 2016. Almacenamiento de carbono en el suelo en dos tipos de sistemas agroforestales cacao (Theobroma cacao L.) y café (Coffea arabica L.) en Hermilio Valdizan (en línea). Tesis Ing. Huánuco, Perú, UNAS. Consultado 21 oct. 2020. Disponible en https://repositorio.unas. edu.pe/handle/UNAS/1363.

Jaramillo, VJ. 2004. El ciclo global del carbono. In Martínez, J; Fernández, A (comps.). Cambio climático: una visión desde México. México DF, México, INE. p. 77-86. Consultado 21 oct. 2020. Disponible en www.data.sedema.cdmx. gob.mx/cambioclimaticocdmx/images/biblioteca cc/Cambio-climatico-una-vision-desde-Mexico-(Julia-Martinez-y-Adrian-Fernandez-Bremauntz-compilado.pdf.

Jiménez, E. 2019. Estimación de la cantidad de carbono almacenado en un agroecosistema de cacao (Theobroma cacao L.) en el sector Shupishiña-San Martín (en línea). Tarapoto, Perú, UNSM. Consultado 21 oct. 2020. Disponible en http://repositorio.unsm.edu.pe/ handle/11458/3506.

Jobbágy, EG; Jackson, RB. 2000. The vertical distribution of soil organic carbon and its relation to climate and vegetation. Ecological applications 10(2):423-436. DOI: https://doi. org/10.1890/1051-0761(2000)010[0423:TVDOSO $] 2.0 . \mathrm{CO} ; 2$.

Kirby, KR; Potvin, C. 2007. Variation in carbon storage among tree species: implications for the management of a small-scale carbon sink Project. Forest Ecology and Management 246(23):208-221. DOI: https://doi.org/10.1016/j. foreco.2007.03.072.

Lizarbe, AM. 2013. Captura de carbono en el sistema agroforestal de Theobroma cacao (cacao), en el distrito de lrazola, región Ucayali (en línea). Tesis Ing. Ucayali, Perú, UNU. 139 p. Consultado 21 oct. 2020. Disponible en http:// repositorio.unu.edu.pe/handle/UNU/2317.

López, KD. 2014. Determinación de la disponibilidad de carbono según la tipificación de los sistemas agroforestales de café en las sub 
cuencas del Río Yuracyacu y Yanayacu. Tesis Ing. Moyobamba, Perú, UNSM. 154 p.

MINAM (Ministerio del Medio Ambiente, Perú). 2009. Mapa de deforestación de la Amazonía peruana 2000 (en línea). Lima, Perú. 22 p. Consultado 21 oct. 2020. Disponible en https:// sinia.minam.gob.pe/documentos/mapadeforestacion-amazonia-peruana-2000.

MINAM (Ministerio del Medio Ambiente, Perú). 2015. Cuantificación y análisis de la deforestación en la Amazonia peruana en el período 2010-2011-2013-2014 (en línea). Lima, Perú. 107 p. Consultado 21 oct. 2020. Disponible en http://infobosques.com/portal/ wpcontent/uploads/2017/03/Memoria Descriptiva Cambios Cobertura Bosque 2014. pdf.

MINAM (Ministerio del Medio Ambiente, Perú). 2016. Ambiente en acción (en línea). Lima, Perú. s.p. Consultado 21 oct. 2020. Disponible en http://www.minam.gob. pe/wp-content/uploads/2016/07/AMBIENTE-ENACCIO\%CC\%81N version-NegraPata-impresion.pdf.

Montagnini, F; Nair, PKR. 2004. Carbon sequestration: an underexploited environmental benefit of agroforestry systems. Agroforestry Systems 61(281):281- 295. DOI: https://doi. org/10.1023/B:AGFO.0000029005.92691.79.

Nair, PKR. 1993. An introduction to agroforestry. Dordrecht, Países Bajos, Kluwer. 437 p. DOI: https://doi.org/10.4236/as.2011.22011.

Nair, PKR. 2004. Agroforestry: trees in support of sustainable agriculture. In Hillel, H; Rosenzweig, C; Powlson, D; Scow, K; Singer, M; Sparks, $\mathrm{D}$ (eds.). Encyclopedia of soils in the environment. Londres, Reino Unido, Elsevier. p. 35-44.

Nair, PKR. 2011. Agroforestry systems and environmental quality: introduction. Journal of Environmental Quality 40(3):784-790. DOI: https://doi.org/10.2134/jeq2011.0076.

Nair, PKR; Fernandes, E. 1984. Agroforestry as an alternative to shifting cultivation (en línea). s.l., ICRAF. Consultado 21 oct. 2020. Disponible en http://www.fao.org/3/aar128e. pdf\#page $=177$.
Nair, PKR; Garrity, D (eds.). 2012. Agroforestry research and development: the way forward (en línea). In Agroforestry-the future of global land use. Nueva York, Estados Unidos de América, Springer. p. 515-531. Consultado 21 oct. 2020. Disponible en https://www.springer.com/gp/ book/9789400746756.

Nair, PKR; Mohan Kumar, B; Nair, VD. 2009. Agroforestry as a strategy for carbon sequestration. Journal of Plant Nutrition and Soil Science 172(1):10-23. DOI: https://doi.org/10.1002/ jpln.200800030.

Odar, BA. 2018. Evaluación de almacenamiento de carbono en sistemas agroforestales de café (Coffea spp.) en el anexo de Vilaya, distrito de Colcamar, provincia de Luya, Amazonas, 2017-2018. Tesis Ing. Chachapoyas, Perú, UNTRM. 58 p.

Oelbermann, M; Voroney, RP; Gordon, AM. 2004. Carbon sequestration in tropical and temperate agroforestry systems: a review with examples from Costa Rica and southern Canada (en línea). Agriculture, Ecosystems \& Environment 104(3):359-377. Consultado 21 oct. 2020. Disponible en https:// agris.fao.org/agris-search/search.do?recor$\underline{\mathrm{dID}=\mathrm{US} 201300968402 \text {. }}$

Osorio, JE. 2015. Almacenamiento y tasas de fijación de biomasa y carbono en diferentes niveles altitudinales en sistemas agroforestales de cacao, distrito de José Crespo y Castillo (en línea). Huánuco, Perú, UNAS. Consultado 21 oct. 2020. Disponible en http://repositorio. unas.edu.pe/handle/UNAS/1086.

Padoch, C; Chota Inuma, J; De Jong, W; Unruh, J. 1985. Amazonian agroforestry: a market-oriented system in Peru. Agroforestry Systems 3(1):47-58. DOI: https://doi.org/10.1007/ BF00045738.

Palm, C; Tomich, T; Van Noordwijk, M; Vosti, S; Gockowski, J; Alegre, J; Verchot, L. 2004. Mitigating GHG emissions in the humid tropics: case studies from the Alternatives to Slash-andBurn Program (ASB) (en línea). Environment, Development and Sustainability 6(1):145162. Consultado 21 oct. 2020. Disponible en 
https://www.asb.cgiar.org/publication/mitigating-ghg-emissions-humid-tropics-case-studies-alternatives-slash-and-burn-program.

Pandey, DN. 2002. Carbon sequestration in agroforestry systems (en línea). Climate Policy 2(4):367-377. Consultado 21 oct. 2020. Disponible en https://www.tandfonline.com/doi/ abs/10.3763/cpol.2002.0240.

Petit-Aldana, J; Uribe-Valle, G; Casanova-Lugo, F; Solorio-Sánchez, J; Ramírez-Áviles, L. 2012. Descomposición y liberación de nitrógeno y materia orgánica en hojas de Leucaena leucocephala (Lam.) de Wit, Guazuma ulmifolia Lam. y Moringa oleifera Lam. en un banco mixto de forraje. Revista Chapingo (Serie ciencias forestales y del ambiente) 18(1):5-25. DOI: https://doi.org/10.5154/r.rchscfa.2011.03.025.

Pocomucha, V; Alegre, J; Abregú, L. 2016. Análisis socio económico y carbono almacenado en sistemas agroforestales de cacao (Theobroma cacao L.) en Huánuco. Ecología Aplicada 15(2):107-114. DOI: http://dx.doi. org/10.21704/rea.v15i2.750.

Ramirez, CA; Panduro, G; Miranda, E. 2014. Captura de carbono en un sistema agroforestal con Theobroma cacao en el campus de la Universidad Nacional De Ucayali, Pucallpa-Perú. TZHOECOEN 6(2):165-180. Consultado 21 oct. 2020. Disponible en http://revistas.uss.edu. pe/index.php/tzh/article/view/11.

Roshetko, JM; Delaney, M; Hairiah, K; Purnomosidhi, P. 2002. Carbon stocks in Indonesian homegarden systems: Can smallholder systems be targeted for increased carbon storage? American Journal of Alternative Agriculture 17(3):138-148. DOI: https://doi.org/10.1079/ AJAA200116.

Sanchez, PA. 2000. Linking climate change research with food security and poverty reduction in the tropics (en línea). Agriculture, Ecosystems \& Environment 82(1-3):371-383. Consultado 21 oct. 2020. Disponible en http:// citeseerx.ist.psu.edu/viewdoc/download?$\underline{\mathrm{doi}=10.1 .1 .521 .217 \& \mathrm{rep}=\mathrm{rep} 1 \& \text { type }=\mathrm{pdf}}$.

Sanchez, PA; Palm, CA; Smyth, TJ. 1990. Approaches to mitigate tropical deforestation by sustainable soil management practices. In Scharpenseel, HW; Schomaker, M; Ayoub, A (eds.). Soils on a warmer earth: effects of expected climate change on soil processes, with emphasis on the tropical and sub-tropics. Nairobi, Kenya, UNEP. p. 211-220. DOI: https:// doi.org/10.1016/S0166-2481(08)70496-3.

Schroth, G; Sinclair, FL (eds.). 2003. Trees, crops, and soil fertility: concepts and research methods. Wallingford, Reino Unido, CABI. 448 p. DOI: https://doi.org/10.1111/j.13652389.2004.0635l.x.

Sifuentes, VC. 2015. Carbono almacenado y capturado en la biomasa aérea en tres sistemas agroforestales (SAF) en Saipai, Santa Lucía. Tingo María, Perú, UNAS.

Sendeco2. 2021. Precios $\mathrm{CO}_{2}$ (en línea, sitio web). Consultado 15 mar. 2021. Disponible en https://www.sendeco2.com/es/precios-co2.

Shibu, J. 2009. Agroforestry for ecosystem services and environmental benefits: an overview. Agroforestry systems (76):1-10. DOI: https:// doi.org/10.1007/s10457-009-9229-7.

Tscharntke, T; Clough, Y; Bhagwat, SA; Buchori, D; Faust, H; Hertel, D; Hölscher, D; Juhrbandt, J; Kessler, M; Perfecto, I; Scherber, C; Schroth, G; Veldkamp, E; Wanger, TC. 2011. Multifunctional shade-tree management in tropical agroforestry landscapes - a review. Journal of Applied Ecology 48(3):619629. DOI: https://doi.org/10.1111/j.13652664.2010.01939.x.

Vilchez, E. 2014. Cuantificación de biomasa y carbono secuestrado en un sistema agroforestal de cacao (Theobroma cacao L.) en Tarapoto - San Martin (en línea). Tesis Ing. Tarapoto, Perú, UNSM. Cosultado 21 oct. 2020. Disponible en http://repositorio.unsm.edu.pe/ handle/11458/657.

Wiersum, KF. 1990. Planning agroforestry for sustainable land use (en línea). In Budd, WW; Duchhart, I; Hardesty, LN; Steiner, F (eds.). Planning for agroforestry. Ámsterdam, Países Bajos, Elsevier Science Publishers. p. 18-32. Cosultado 21 oct. 2020. Disponible en https:// library.wur.nl/WebQuery/titel/803488. 
Winterbottom, R; Hazlewood, PT. 1987. Agroforestry and sustainable development: making the connection. Ambio 16(2-3): 100110.

Young, A. 1997. Agroforestry for soil management. 2 ed. Nairobi, Kenya, ICRAF. 320 p.

Zavala, W; Merino, E; Peláez, P. 2018. Influencia de tres sistemas agroforestales del cultivo de cacao en la captura y almacenamiento de carbono. Scientia Agropecuaria 9(4):493-501. 